\title{
VELOCITY DISTRIBUTIONS IN THE FLOW OF SOLID PARTICLES IN AN INCLINED OPEN CHANNEL
}

\author{
MASARU ISHIDA AND TAKASHI SHIRAI \\ Research Laboratory of Resources Utilization, \\ Tokyo Institute of Technology, Yokoyama 227
}

\begin{abstract}
The velocity of solid particles flowing in an inclined open channel of which the bottom plate was covered with very rough sandpaper was measured for three kinds of particle.

Except for the region near the bottom plate, the velocity distribution normal to the bottom plate appeared to be linear. The velocity gradient in that region was almost independent of the thickness of the particle layer and increased as the slope of the channel became steep.

These observed velocity distributions were analyzed based on the variational principle, by which the velocity distribution could be obtained as the solution which minimized a certain integral consisting of several energy terms. It was found that such analysis could explain the main feature of the particle flow in an inclined channel, and the following relation between stress and rate of deformation was obtained.

$$
\tau_{y z}=\boldsymbol{k}_{\tau} \boldsymbol{y}-\boldsymbol{k}_{\mu} \boldsymbol{y}\left(\boldsymbol{d} \boldsymbol{v}_{z} / \boldsymbol{d y}\right)
$$
\end{abstract}

It was also found that the critical inclination angle of the channel, which was anticipated by the analysis, corresponded to the angle of repose.

\section{Introduction}

The gravity flow of bulk solid particles in an inclined open channel has been investigated on the basis of observation at the free surface and at the side and bottom surfaces adjacent to transparent walls ${ }^{4,5,9)}$. And it was shown that the particle velocity was greatest at the free surface, decreased with depth, and was smallest at the bottom.

Hence, this flow is relatively simple and gives a very thick deformation zone, i.e., the zone with velocity gradient so that one can measure the velocity distribution in it minutely. Consequently, this flow system is quite suitable for examining rheological properties of solid particles.

In the present paper, the velocity within the granular layer was measured in detail and the velocity distributions obtained were analyzed on the basis of the variational principle by comparing them with those for fluids. A simple relation between stress and rate of deformation for this particle flow was proposed.

\section{Experimental}

\section{1 Apparatus}

Figure 1 shows the outline of the apparatus used in this study. The inclined channel (4) was $22 \mathrm{~mm}$ wide and $850 \mathrm{~mm}$ long. To make the flow in it similar to that on an inclined flat plate, the side walls (4-a) were made of smooth glass plate. Moreover, the bot-

\footnotetext{
Received May 6, 1978. Correspondence concerning this article should be addressed to M. Ishida.
}

tom plate (4-b) was covered with very rough sandpaper, $\# 40$, to prevent the particles from sliding on it.

The upstream end of this main channel was connected to a guide channel (3) which was slightly steeper than the main one. It was installed to accelerate the particle velocity to some extent beforehand and hence to minimize the velocity change along the longitudinal direction in the main channel.

A hopper (1) which could store $30 l$ of particles was located above the guide channel. A nozzle (2) was provided at the bottom of the hopper to control the flow rate of the particles.

\section{2 Procedure}

First set the slope of the channels, select proper nozzle size, and charge the particles into the hopper. Then, start feeding the channel with the particles. After a steady flow is attained, measure the velocity

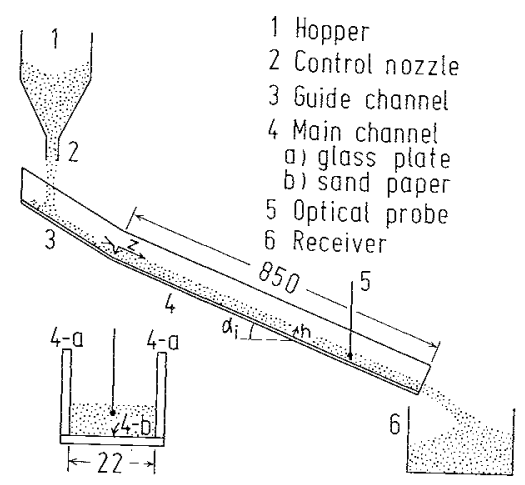

Fig. 1 Outline of experimental apparatus 
Table 1 Properties of solid particles

\begin{tabular}{lcccccc}
\multicolumn{1}{c}{ Particles } & $\begin{array}{c}d_{p} \\
{[\mathrm{~mm}]}\end{array}$ & $\begin{array}{c}\rho_{B} \\
{\left[\mathrm{~g} / \mathrm{cm}^{3}\right]}\end{array}$ & $\begin{array}{c}\phi_{\tau} \\
{[\mathrm{deg}]}\end{array}$ & $\begin{array}{c}\alpha_{i_{c}} \\
{[\mathrm{deg}]}\end{array}$ & $\begin{array}{c}k_{\tau} \\
{\left[\mathrm{g} / \mathrm{cm}^{2} \cdot \mathrm{sec}^{2}\right]}\end{array}$ & $\begin{array}{c}k_{\mu} \\
{\left[\mathrm{g} / \mathrm{cm}^{2} \cdot \mathrm{sec}^{2}\right.}\end{array}$ \\
\hline Glass beads & $0.35-0.50$ & 1.50 & 23 & 23.0 & $5.7 \times 10^{2}$ & 0.62 \\
Porous alumina & $0.21-0.30$ & 0.95 & 31 & 31.1 & $4.8 \times 10^{2}$ & 0.62 \\
F. C. C. & $0.044-0.063$ & 0.80 & 32 & 32.4 & $4.2 \times 10^{2}$ & 0.30 \\
\hline
\end{tabular}

distribution within the particle layer. A fine optical fiber probe which was the same as that used in the previous study ${ }^{7}$ was used to measure the particle velocity at each local point within the layer. Most measurement was performed $650 \mathrm{~mm}$ from the upstream end of the main channel.

Three kinds of particle were used in this study. Their size $d_{p}$, bulk density $\rho_{B}$, and angle of repose $\phi_{r}$ are shown in Table 1.

\subsection{Results}

Figure 2 shows the effect of the side walls on the lateral particle velocity distribution at $z=650 \mathrm{~mm}$. The particle velocity near the side glass walls was found to be a little smaller than that at the center of the channel. However, since the difference between them was small, the data presented in this paper were all taken at the center of the channel.

Figure 3 shows the relation between particle velocity, $v_{z}$, and height from the bottom plate, $h$, for various inclination angles of the channel, $\alpha_{i}$. When $\alpha_{i}$ was small, the particle velocity distribution was composed of two parts. In the lower part near the bottom rough surface the particle velocity was low and increased only slightly with height. On the other hand, in the upper part near the free surface it increased almost linearly with height. As the inclination angle of the channel $\alpha_{i}$ was increased the region of the lower part became thin, and the velocity distribution is described by the following simple relation.

$$
v_{z}=c \cdot h \quad(c: \text { constant })
$$

Figure 4 shows similar relations for thicker layers. By comparing Fig. 3 with Fig. 4, it was found that the velocity gradient in the upper region with linear velocity distribution was almost independent of layer thickness for such thin layers.

The velocity gradient in the upper region with linear velocity distribution was calculated from the data in Fig. 3 and is plotted against the slope of the channel, $\sin \alpha_{i}$, by open circles in Fig. 5 . It was found that the velocity gradient in that region increased almost linearly with $\sin \alpha_{i}$.

So far, only the data for the glass beads were examined. For the other particles, similar results were also obtained. The relation between slope of the channel and velocity gradient in the linear velocity distribution region are shown also in Fig. 5.

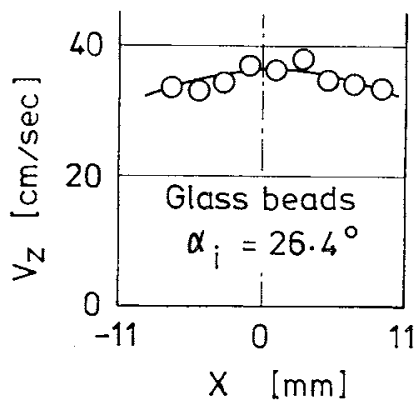

Fig. 2 Example data for lateral distribution of particle velocity

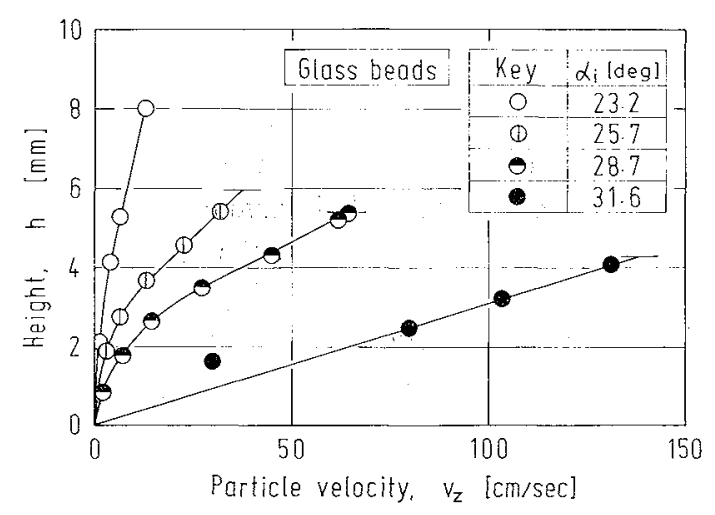

Fig. 3 Effect of inclination angle of the channel, $\alpha_{i}$, on $v_{z}-h$ relation for thinner layers

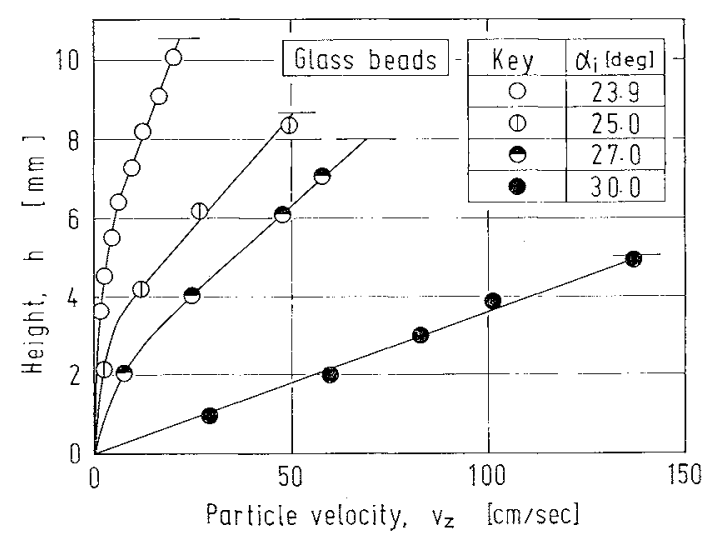

Fig. 4 Effect of inclination angle of the channel, $a_{i}$, on $v_{z}-h$ relation for thicker layers

\section{Variational Analysis}

When these observed velocity distributions are compared with those of fluids, one may find out several distinctive characteristics of the particle flow. Espe- 


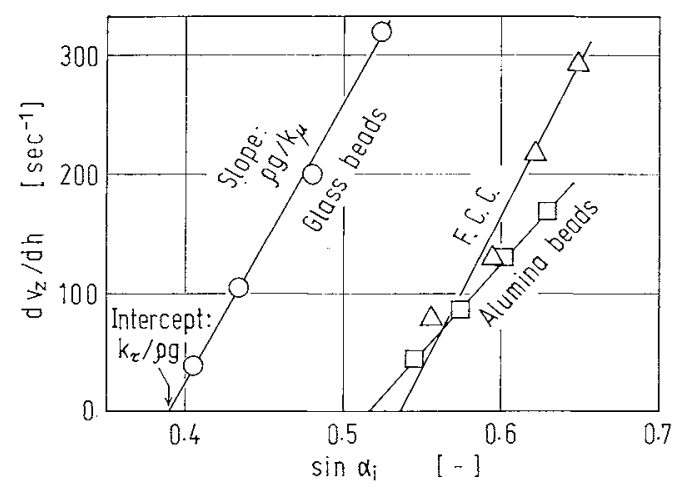

Fig. 5 Relation between the slope of the channel and the velocity gradient for three kinds of particle

cially, it is noticed that the velocity gradient at the free surface was not zero. Such peculiarity in particle flow has been observed also for the case when a horizontal disk was rotated within a particle bed ${ }^{7}$.

Although the size of particles is much greater than that of fluid molecules, a throng of particles may be treated macroscopically as a continuum, and the equation of continuity and the stress equation of motion can be applied. However, they are not sufficient to describe the motion of matter under given boundary conditions. In addition, one must state the relation between stress and deformation, in order to describe the behavior of the particular matter to be considered. For a Newtonian fluid, this relation gives the definition of viscosity. Also for a non-Newtonian fluid, several relations such as Bingham model, Ostwald-de Waale model, and Reiner-Philipoff model have been proposed ${ }^{11}$. For the motion of particles, however, such relations have not been suggested.

Consequently, an attempt was made to find such a relation for particle flow based on the experimental results by applying the variational principle ${ }^{2,3)}$.

The parabolic velocity distribution for a Newtonian fluid on an inclined flat plate can be obtained by finding the velocity distribution to minimize the following integral.

$$
J=\int_{0}^{H}\left[\frac{\mu}{2}\left(\frac{d v_{z}}{d h}\right)^{2}-\rho g v_{z} \sin \alpha_{i}\right] d h
$$

The integrand consists of two energy terms: The first one is viscous dissipation, $(1 / 2)(-\tau: \nabla v)$, and the second one is rate of work done by gravity on the volume element, $\boldsymbol{\rho}(\boldsymbol{v} \cdot \boldsymbol{g})^{11}$.

The velocity distribution to minimize the value of $J$ can be found by Pontryagin's maximum principle ${ }^{6,8}$, as summarized in Appendix, and the following parabolic distribution is obtained.

$$
v_{z}=\frac{\rho g H^{2} \sin \alpha_{i}}{2 \mu}\left[1-\left(\frac{y}{H}\right)^{2}\right]
$$

Similarly, the velocity distribution for a Bingham fluid on an inclined flat plate can be obtained by finding the velocity distribution to minimize the following integral.

$$
J=\int_{0}^{H}\left[\tau_{B} \frac{d v_{z}}{d h}+\frac{\mu_{B}}{2}\left(\frac{d v_{z}}{d h}\right)^{2}-\rho g v_{z} \sin \alpha_{i}\right] d h
$$

The numerical coefficient of the first term in the integrand is twice that of the second term, because these terms are derived from ${ }^{2}$

$$
\int_{0}^{\left(d v_{z} / d h\right)}\left[\tau_{B}+\mu_{B}\left(d v_{z} / d h\right)\right] d\left(d v_{z} / d h\right)
$$

And the following well-known relation can be obtained as outlined in Appendix.

$$
\begin{array}{ll}
v_{z}=\frac{\rho g\left(H-H_{c}\right)^{2} \sin \alpha_{i}}{2 \mu_{B}} & \text { for } y<H_{c} \\
v_{z}=\frac{\rho g\left(H-H_{c}\right)^{2} \sin \alpha_{i}}{2 \mu_{B}}\left[1-\left(\frac{y-H_{c}}{H-H_{c}}\right)^{2}\right] & \text { for } y \geqq H_{c}
\end{array}
$$

where the critical thickness for a Bingham fluid is given by

$$
H_{c}=\tau_{B} /\left(\rho g \sin \alpha_{i}\right)
$$

Meanwhile, the shear stress $\tau_{y z}$ at depth $y$ is given by the following equation:

$$
\tau_{y z}=\rho g y \sin \alpha_{i}
$$

By combining Eq. (9) and Eqs. (3) and (7), one obtains the following relation between stress and rate of deformation:

$$
\begin{array}{ll}
\tau_{y z}=-\mu\left(d v_{z} / d y\right) & \text { for a Newtonian fluid } \\
\tau_{y z}=\tau_{B}-\mu_{B}\left(d v_{z} / d y\right) & \text { for a Bingham fluid }
\end{array}
$$

Therefore it may be induced that the velocity distribution for particle flow on an inclined flat plate can be obtained also by minimizing a certain integral. As a first-step approximation, the linear distribution given by Eq. (1) is assumed and the following integral which explains it is proposed.

$$
J=\int_{0}^{H}\left[k_{\tau} y\left(\frac{d v_{z}}{d h}\right)+\frac{k_{\mu} y}{2}\left(\frac{d v_{z}}{d h}\right)^{2}-\rho g v_{z} \sin \alpha_{i}\right] d h
$$

where $k_{\tau}$ and $k_{\mu}$ are constants.

Then the following velocity distribution can be obtained, as outlined in Appendix.

$$
\begin{array}{ll}
v_{z}=0 & \text { for } \alpha_{i}<\alpha_{i_{c}} \\
v_{z}=\frac{\rho g \sin \alpha_{i}-k_{\tau} \cdot h}{k_{p}} & \text { for } \alpha_{i} \geqq \alpha_{i_{c}}
\end{array}
$$

where the critical angle $\alpha_{i_{\mathrm{c}}}$ is given by

$$
\sin \alpha_{i_{c}}=k_{\tau} /(\rho g)
$$

Equation (14) shows that the velocity gradient is independent of layer thickness $H$.

By combining Eq. (9) and Eq. (14), one obtains the following relation between stress and rate of deformation for the flow of particles on an inclined plate.

$$
\tau_{y z}=k_{\tau} y-k_{\mu} y\left(d v_{z} / d y\right)
$$

Equation (16) should be considered as a simple model which can explain the main features of particle 
flow in an inclined channel. Much more effort should be made to establish a more general relation which is applicable to various kinds of flow of solid particles.

\section{Discussion}

Although Eqs. (12) and (16), respectively, are very similar to Eqs. (4) and (11) for a Bingham fluid, both yield stress $k_{\tau} y$ and apparent viscosity $k_{\mu} y$ for particles are proportional to $y$. This may be related closely to the fact that buoyancy does not act in the layer of solid particles. This depth $y$, which is proportional to the normal strees $\sigma$, plays a very important role and gives a velocity distribution which is entirely different from that for a Bingham fluid.

For example, a Bingham fluid is able to flow even at a small inclination angle $\alpha_{i}$ when the thickness of the layer is greater than critical thickness $H_{c}$. For particles, however, when $\alpha_{i}$ is less than critical inclination angle $\alpha_{i_{c}}$, the particle layer remains stationary.

The critical inclination angle $\alpha_{i_{c}}$ for each kind of particle can be obtained experimentally from the intercept on the abscissa in Fig. 5. As shown in Table 1, the obtained critical inclination angle $\alpha_{i_{c}}$ is almost equal to the angle of repose $\phi_{r}$. Consequently, the critical inclination angle $\alpha_{i_{c}}$ anticipated by variational analysis may be considered to correspond to the angle of repose.

Finally, the values of $k_{\tau}$ and $k_{\mu}$ for each kind of particle are obtained from the critical inclination angle and the slope of the straight line in Fig. 5, by substituting the bulk density at stationary state, $\rho_{B}$, for $\rho$ in Eqs. (15) and (14) as a first-step approximation. They are shown in Table 1.

These values of $k_{\tau}$ and $k_{k}$ may be considered as flow properties of each kind of particle. But, as has been stated before, further work is required to grasp all aspects of particle flow.

\section{Conclusion}

The velocity distribution within the layer of partiples flowing in an inclined channel was measured and the following conclusions were obtained.

1. The velocity distribution near the free surface appeared to be linear and the velocity gradient in that region was independent of the layer thickness.

2. The velocity gradient increased as the inclination angle of the channel became greater.

3. Main features of these linear velocity distributions were explained by variational analysis.

4. The critical inclination angle anticipated by variational analysis was found to correspond to the angle of repose.

Appendix: Velocity Distributions to Make the Intergral $J$ Minimum

\section{Newtonian fluid}

The state equation for this flow system is

$$
d v_{z} / d h=p \quad(p \geqq 0)
$$

Then, try to find $p$ which will maximize the following Hamiltonian function $H$.

$$
\begin{aligned}
H & =-\left[(\mu / 2) p^{2}-\rho g v_{z} \sin \alpha_{i}\right]+\psi_{\mathrm{i}}[p] \\
& =-\frac{\mu}{2}\left(p-\frac{\phi_{1}}{\mu}\right)^{2}+\frac{\phi_{1}^{2}}{2 \mu}+\rho g v_{z} \sin \alpha_{i}
\end{aligned}
$$

where $\phi_{1}$ is a multiplier and satisfies the following equation.

$$
\frac{d \phi_{1}}{d h}=-\frac{\partial H}{\partial v_{z}}=-\rho g \sin \alpha_{i}
$$

Hence,

$$
\phi_{1}=-\rho g h \sin \alpha_{i}+c_{1}
$$

$H$ becomes maximum when

$$
p \equiv \frac{d v_{z}}{d h}=\frac{\psi_{1}}{\mu}=-\frac{-\rho h \sin \alpha_{i}+c_{1}}{\mu}
$$

Hence,

$$
v_{z}=\left(-\rho g h^{2} \sin \alpha_{i}+2 c_{1} h\right) / 2 \mu+c_{2}
$$

The integral constants $c_{1}$ and $c_{2}$ are determined by

$$
\begin{array}{ll}
v_{z}=0 & \text { at } h=0 \\
\psi_{1}=0 & \text { at } h=H
\end{array}
$$

Equation (A-5) holds because the velocity $v_{z}$ at $h=H$ is not given. Then, Eq. (3) is obtained. Note that the condition, $d v_{z} / d h=0$ at $h=H$, has not been used to derive the velocity distribution.

\section{Bingham fluid}

Hamiltonian function $H$ is given as

$$
\begin{aligned}
\bar{H} & =-\left[\tau_{B} p+\left(\mu_{B} / 2\right) p^{2}-\rho g v_{z} \sin \alpha_{i}\right]+\phi_{1}[p] \\
& =-\frac{\mu_{B}}{2}\left(p-\frac{\phi_{1}-\tau_{B}}{\mu_{B}}\right)^{2}+\frac{\left(\phi_{1}-\tau_{B}\right)^{2}}{2 \mu_{B}}+\rho g v_{z} \sin \alpha_{i}
\end{aligned}
$$

Hence, Eqs. (A-2), (A-3), and (A-5) hold also in this case. Since $p$ must be zero or positive, the following two cases are considered.

$$
\begin{aligned}
& \text { (Case 1) } \phi_{1}-\tau_{B}<0 \quad \therefore y<H_{c} \\
& p \equiv d v_{z} / d h=0 \\
& p \equiv \frac{d v_{z}}{d h}=\begin{array}{c}
\phi_{\perp}-\tau_{B} \\
\mu_{B}
\end{array}=\frac{\rho g \sin \alpha_{i}(H-h)-\tau_{B}}{\mu_{B}}
\end{aligned}
$$

Equation (7) is obtained by integrating Eq. (A-6) with the boundary condition, Eq. (A-4).

\section{Particles}

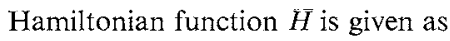

$$
\begin{aligned}
H= & -\left[k_{\tau}(H-h) p+\left(k_{\mu} / 2\right)(H-h) p^{2}-\rho g v_{z} \sin \alpha_{i}\right]+\psi_{1}[p]+\psi_{2} \\
= & -k_{\mu}(H-h)\left[p-\frac{\psi_{1}-k_{\tau}(H-h)}{k_{\mu}(H-h)}\right]^{2}+\frac{\left[\psi_{1}-k_{\tau}(H-h)\right]^{2}}{2 k_{\mu}(H-h)} \\
& +\rho g v_{z} \sin \alpha_{i}+\phi_{2}
\end{aligned}
$$

Hence, Eqs. (A-2), (A-3), and (A-5) hold also in this case.

$$
\begin{array}{r}
\text { (Case 1) } \phi_{1}-k_{\tau}(H-h)<0 \quad \therefore \sin \alpha_{i}<k_{\tau} /(\rho g) \\
p \equiv d v_{z} / d h=0 \quad \therefore v_{z}=0 \\
\left(\text { Case 2) } \phi_{1}-k_{\tau}(H-h) \geqq 0 \quad \sin \alpha_{i} \geqq k_{\tau} /(\rho g)\right. \\
p \equiv \frac{d v_{z}}{d h}=\frac{\left(\rho g \sin \alpha_{i}-k_{\tau}\right)(H-h)}{k_{\mu}(H-h)}
\end{array}
$$

Equation (14) is obtained by integrating Eq. (A-7) with the boundary condition, Eq. (A-4).

\section{Acknowledgment}

The authors wish to thank Mr. Shojiro Kobayashi, Mr. 
Haruhiko Sato, and Mr. Koji Ogawa for their help in performing the experimental work.

\section{Nomenclature}

$d_{p} \quad=$ diameter of particles

$H \quad=$ thickness of the layer

$H_{c} \quad=$ critical thickness for a Bingham fluid

$h \quad=$ height measured from the bottom plate

$k_{\mu} \quad=$ coefficient for apparent viscosity of particles

$k_{\tau} \quad=$ coefficient for yield stress of particles

$v_{z} \quad=$ longitudinal velocity

$=$ lateral distance

$=$ depth measured from the free surface [mm], [cm]

$=$ longitudinal distance

$[\mathrm{mm}],[\mathrm{cm}]$

$\alpha_{i} \quad=$ inclination angle of the channel [deg]

$\alpha_{i_{c}}=$ critical inclination angle of the channel [deg]

$\mu \quad=$ viscosity $\quad[\mathrm{g} / \mathrm{cm} \cdot \mathrm{sec}]$
$\mu_{B}$ $=$ apparent viscosity for a Bingham fluid

$\rho \quad=$ density

$\tau_{B} \quad=$ yield stress for a Bingham fluid

$\tau_{y z} \quad=$ shear stress

$\phi_{r} \quad=$ angle of repose

\section{Literature Cited}

1) Bird, R. B., W. E. Stewart, and E. N. Lightfoot: "Transport Phenomena", John Wiley \& Sons, Inc. (1960).

2) Johnson, M. W. Jr.: Phys. Fluids, 3, 871 (1960).

3) Lamb, H.: "Hydrodynamics", 6th ed., p. 617, Dover Publications (1932).

4) Ohyama, Y.: Riken Iho, 19, 1052 (1940).

5) Ono, E.: Ohyo Butsuri, 36, 347 (1967).

6) Pontryagin, L. S., et al.: "The Mathematical Theory of Optimal Processes", Interscience Publishers, Inc. (1962).

7) Shirai, T., M. Ishida, Y. Ito, N. Inoue and S. Kobayashi: J. Chem. Eng. Japan, 10, 40 (1977).

8) Uno, T. and T. Kikuchi: "Saidai Genri Nyumon", Kyoritsu Shuppan (1967).

9) Utsumi, R. and K. Ueda: Zairyo, 20, 773 (1971). 